\title{
A contrast agent recognizing activated platelets reveals murine cerebral malaria pathology undetectable by conventional MRI
}

\author{
Constantin von zur Muhlen, ${ }^{1,2}$ Nicola R. Sibson, ${ }^{3}$ Karlheinz Peter, ${ }^{4}$ \\ Sandra J. Campbell, ${ }^{5}$ Panop Wilainam, ${ }^{5}$ Georges E. Grau, ${ }^{6}$ \\ Christoph Bode, ${ }^{2}$ Robin P. Choudhury, ${ }^{1}$ and Daniel C. Anthony ${ }^{5}$
}

1Department of Cardiovascular Medicine, University of Oxford, Oxford, United Kingdom. 2Division of Cardiology and Angiology, University Hospital Freiburg, Freiburg, Germany. ${ }^{3}$ Department of Physiology, Anatomy, and Genetics, University of Oxford, Oxford, United Kingdom. ${ }^{4}$ Centre for Thrombosis and Myocardial Infarction, Baker Heart Research Institute, Melbourne, Victoria, Australia. ${ }^{5}$ Department of Pharmacology, University of Oxford, Oxford, United Kingdom. ${ }^{6}$ Vascular Immunology Unit, Faculty of Medicine, and Bosch Institute, University of Sydney, Sydney, New South Wales, Australia.

\begin{abstract}
Human and murine cerebral malaria are associated with elevated levels of cytokines in the brain and adherence of platelets to the microvasculature. Here we demonstrated that the accumulation of platelets in the brain microvasculature can be detected with MRI, using what we believe to be a novel contrast agent, at a time when the pathology is undetectable by conventional MRI. Ligand-induced binding sites (LIBS) on activated platelet glycoprotein IIb/IIIa receptors were detected in the brains of malaria-infected mice 6 days after inoculation with Plasmodium berghei using microparticles of iron oxide (MPIOs) conjugated to a single-chain antibody specific for the LIBS (LIBS-MPIO). No binding of the LIBS-MPIO contrast agent was detected in uninfected animals. A combination of LIBS-MPIO MRI, confocal microscopy, and transmission electron microscopy revealed that the proinflammatory cytokine TNF- $\alpha$, but not IL-1 $\beta$ or lymphotoxin- $\alpha$ (LT- $\alpha)$, induced adherence of platelets to cerebrovascular endothelium. Peak platelet adhesion was found $12 \mathrm{~h}$ after TNF- $\alpha$ injection and was readily detected with LIBS-MPIO contrast-enhanced MRI. Temporal studies revealed that the level of MPIO-induced contrast was proportional to the number of platelets bound. Thus, the LIBS-MPIO contrast agent enabled noninvasive detection of otherwise undetectable cerebral pathology by in vivo MRI before the appearance of clinical disease, highlighting the potential of targeted contrast agents for diagnostic, mechanistic, and therapeutic studies.
\end{abstract}

\section{Introduction}

Cerebral malaria (CM) is a major cause of death in tropical and subtropical regions, killing over 3 million individuals worldwide per year (1). Platelet sequestration in the cerebral microvasculature plays a pivotal role in the pathogenesis of CM (2), and antiplatelet therapy has been shown to improve outcome in CM patients $(3,4)$. The histopathology of mice infected with Plasmodium berghei ANKA reveals extensive damage to vascular endothelial cells and plugging of vessels caused by platelet thrombi (5). Similarly, immunohistochemistry for the platelet-specific glycoprotein IIb/IIIa (GPIIb/IIIa) receptor, the activated conformation of which is responsible for platelet linkage via fibrinogen, revealed that platelet accumulation occurs in the microvasculature of patients with CM (6). The mechanisms of platelet aggregation and adhesion are not completely understood. However, the local production of the proinflammatory cytokine TNF may be a contributing factor $(7,8)$. TNF is elevated in postmortem brain tissue of CM patients and in murine models of CM, where it is known to upregulate the expression of the selectins on the cerebral vasculature (9). Other proinflammatory cytokines, such as IL- $1 \beta$ and lymphotoxin- $\alpha$ (LT- $\alpha$ ), are also expressed within the brain parenchyma of mice and humans with

Nonstandard abbreviations used: CM, cerebral malaria; Gd-DTPA, gadopentetic acid; GPIIb/IIIa, glycoprotein IIb/IIIa; LIBS, ligand induced binding site(s); LT- $\alpha$, lymphotoxin- $\alpha ; \mathrm{MPIO}$, microparticle of iron oxide; TE, echo time; TR, repetition time. Conflict of interest: The authors have declared that no conflict of interest exists. Citation for this article: J. Clin. Invest. 118:1198-1207 (2008). doi:10.1172/JCI33314.
$\mathrm{CM}$ and may contribute to the sequestration of platelets by activating the microvasculature (10). However, each of these cytokines gives rise to the recruitment of distinct populations of leukocytes across the intact brain endothelium despite the induction of the same pattern of adhesion molecule expression. The differential induction of chemokines is likely to determine which populations are recruited, but it is not known whether platelet adhesion to the brain microvasculature is dependent on the expression of specific cytokines.

A noninvasive approach for the specific detection of activated platelets or platelet thrombi in the cerebral microvasculature under different conditions of cytokine expression could help in determining the influence of cytokines on vascular platelet adhesion in CM and other neuropathologies. Recent progress in MRI has enabled the detection of such molecular targets by designing contrast agents that bind to cellular receptors or surface antigens $(11,12)$. By delivering high payloads of contrast agent such as iron oxide particles to molecular epitopes, imaging of even sparsely distributed molecules is possible $(13,14)$. We have previously demonstrated that vascular inflammation, such as is found in multiple sclerosis, can be detected in vivo using VCAM-1-targeted microparticles of iron oxide (MPIOs) in conjunction with MRI (15). In addition, we have described the unique possibility of selectively targeting activated platelets using single-chain antibodies that recognize the ligand binding pocket or ligand-induced binding sites (LIBS) of GPIIb/IIIa (16-18). The latter epitope becomes exposed only upon activation through receptor-ligand binding and therefore offers the opportunity to target activated platelets, such as are found on damaged 
A

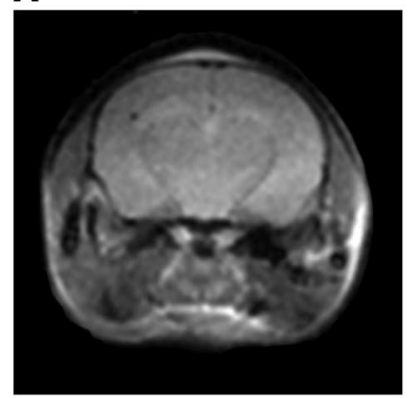

C

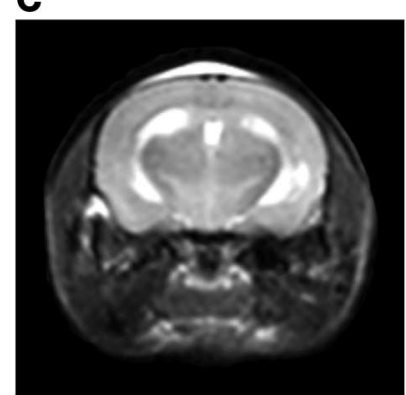

B

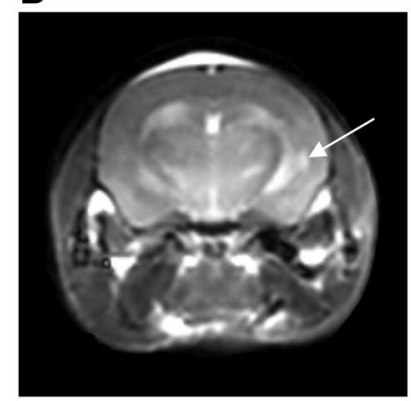

D

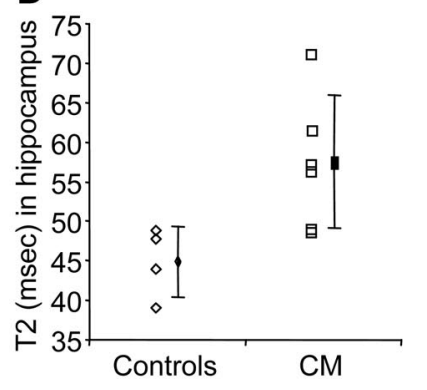

endothelium caused by inflammation or atherosclerotic plaque rupture. By conjugating the LIBS antibody to MPIOs (referred to herein as LIBS-MPIO), we recently demonstrated the possibility of detecting endovascular platelet adhesion by ex vivo MRI (19).

In the present study, we used the above-described activation-specific platelet contrast agent LIBS-MPIO in an animal model to detect vascular platelet aggregation associated with CM before pathology was visible by conventional in vivo MRI. We further demonstrated that platelet accumulation was induced in the brain microvasculature by the proinflammatory cytokine TNF, but not by either IL-1 $\beta$ or LT- $\alpha$. Following platelet accumulation, TNF, but not IL- $1 \beta$, also induced the adherence of mononuclear cells to the cerebral vasculature in a manner very reminiscent of CM pathology.

\section{Results}

Imaging of CM with conventional MRI. Conventional MRI was performed throughout the development of disease in mice infected with the $\mathrm{CM}$ parasite. No abnormalities were detected until day 7 , when breakdown of the blood-brain barrier was evident as hyperintense areas on $\mathrm{T}_{1}$-weighted images obtained after injection of gadopentetic acid (Gd-DTPA) that were not present prior to Gd-DTPA injection (Figure 1, A and B). Discrete hyperintensities were also present on $\mathrm{T}_{2}$-weighted images (Figure $1 \mathrm{C}$ ), which coincided with the Gd-DTPA-enhancing lesions. The evaluation of $\mathrm{T}_{2}$ maps revealed a significant increase in $T_{2}$ within the hippocampus (Figure 1D). However, by day 7 the mice were moribund, and MRI at this time provided little additional information on the pathogenic process.

\section{Figure 2}

In vitro MPIO platelet binding. (A-C) Nonactivated or ADP-activated platelets were incubated with LIBS-MPIO (A), control-MPIO (B), or nonfunctionalized MPIOs (C). Note the presence of LIBS-MPIO binding on the surface of the activated platelets on the thrombus, which was not observed on nonactivated platelets or with the control MPIO or nonfunctionalized MPIO.

\section{Figure 1}

Conventional imaging of murine CM. (A) Coronal $\mathrm{T}_{1}$-weighted image acquired 7 days after the injection of $10^{6} P$. berghei ANKA-parasitized rbcs. (B) $T_{1}$-weighted image from the same animal following injection of Gd-DTPA. Note the regions of increased signal intensity compared with $\mathbf{A}$, indicating blood-brain barrier breakdown (arrow). (C) $\mathrm{T}_{2}$-weighted image of the same slice showing increased signal intensity in the same regions as the Gd-DTPA enhancement. (D) Significant elevation in $T_{2}$ in the hippocampi of day- 7 terminal-stage $C M$ mice $(P<0.05)$.

Before day 7 , no overt clinical signs were evident. Thus our studies using conventional MRI techniques failed to reveal the presence of CNS pathology before the appearance of overt clinical signs.

Imaging of CM using a platelet-specific contrast agent. Murine and human $\mathrm{CM}$ is associated with the adherence of platelets to an intact brain endothelium $(20,21)$. The aim of this study was to determine whether we could distinguish between $\mathrm{CM}$ and control mice at a time when no overt disease was present using a contrast agent that recognizes activated platelets. Our in vitro experiments revealed that the functionalized MPIOs, which recognize the LIBS of GPIIb/IIIa receptors (LIBS-MPIO), bound to ADP-activated platelets alone. No significant binding was observed with MPIOs conjugated to control antibodies (control-MPIO) or nonfunctionalized MPIOs (Figure 2).

When MRI was performed at day 5 after $P$. berghei ANKA infection, some contrast enhancement, in the form of isolated focal hypointensities, was observed on $\mathrm{T}_{2}$-weighted images after LIBS-MPIO injection (Supplemental Figure 1; supplemental material available online with this article; doi:10.1172/JCI33314DS1). However, after injection of LIBS-MPIO in CM-infected mice at day 6, more extensive MPIOassociated hypointensity was evident in and around cortical vessels, delineating areas of LIBS-MPIO binding as demonstrated in 2 representative slices at different levels of the same brain (Figure 3A, arrows). Conversely, CM-infected animals injected with control-MPIO exhibit-
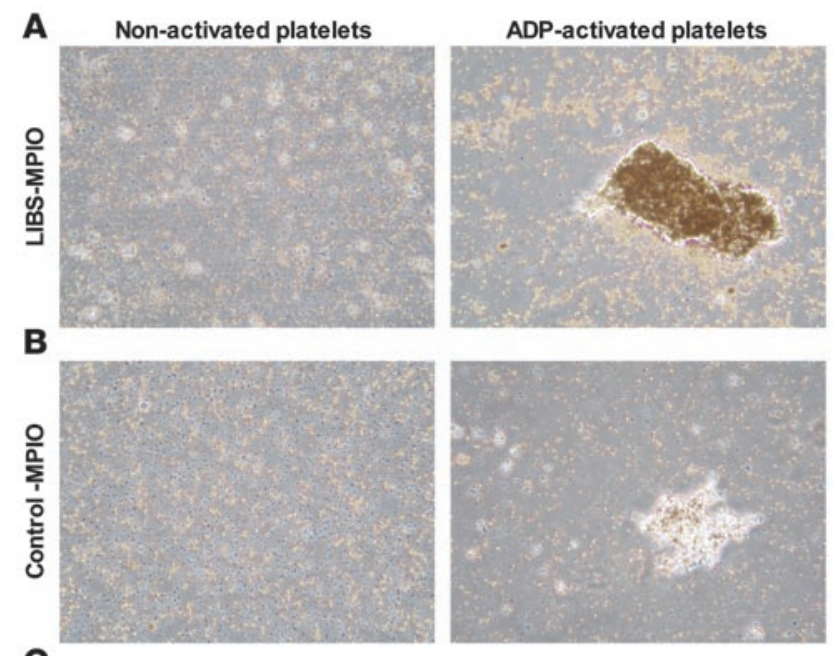

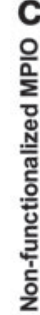
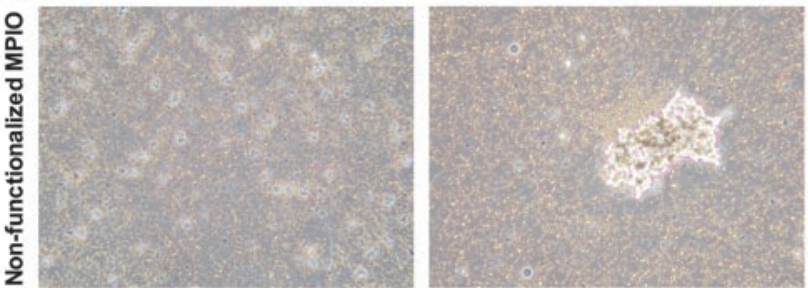
A
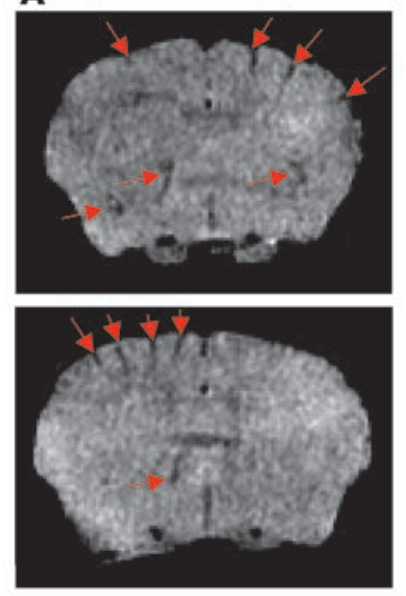

B
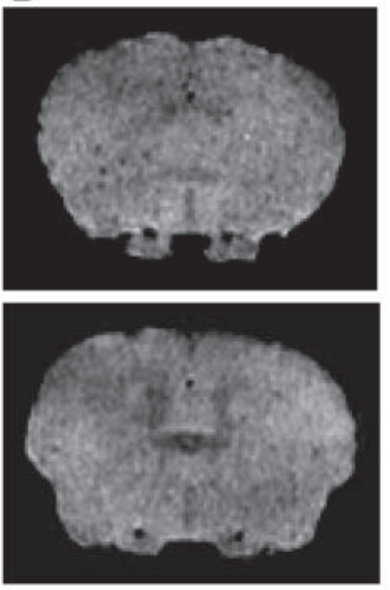

C

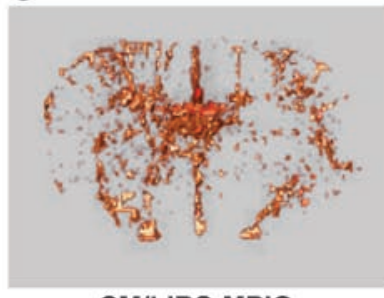

CM/LIBS-MPIO

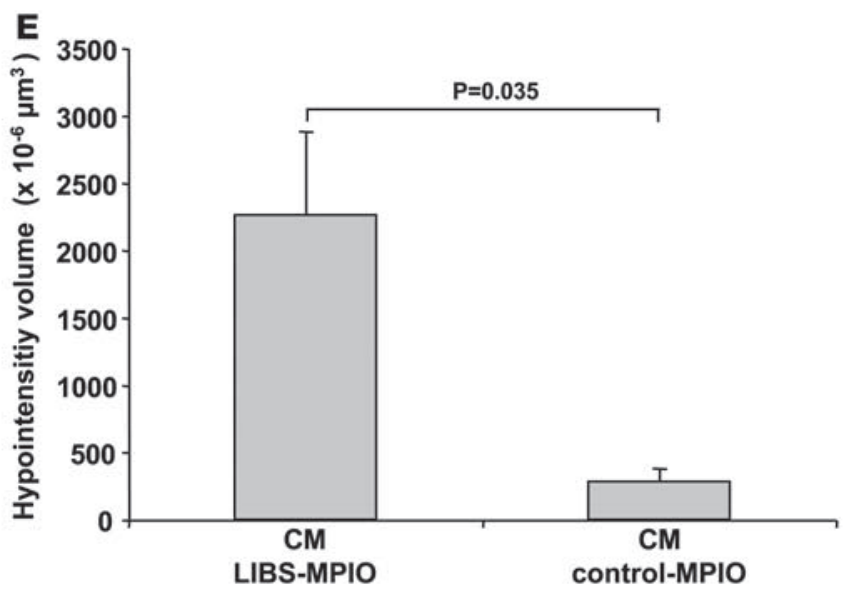

Figure 3

Data from animals with CM and LIBS-MPIO contrast agent injection. (A and B) $T_{2}$-weighted 3D gradient-echo images from CM mice following intravenous injection of LIBS-MPIO (A) and control-MPIO (B) are presented in 2 representative slices at 2 different levels within the same brain. Areas of MPIO-induced signal appeared as dark signal voids (arrows) in cortical regions of the LIBS-MPIO-injected mouse, but not in the controlMPIO-injected mouse. (C and D) 3D reconstruction confirmed the cortical binding pattern in the LIBS-MPIO-injected mouse (C), whereas only modest background binding was evident in the control-MPIO animal (D). (E) Quantification of signal voids demonstrated a significant difference between the LIBS-MPIO- and control-MPIO-injected animals.

ed no negative contrast in corresponding areas (Figure 3B). Using a 3D reconstruction of the original MRI data stack, we observed enhanced binding of LIBS-MPIO in cortical regions of the brain (Figure 3C and Supplemental Movie 1), while injection of control-MPIO did not give rise to specific binding (Figure 3D and Supplemental Movie 2). Using volumetric quantification, a significant increase in the extent of signal voids per volume was confirmed for LIBS-MPIO- compared with control-MPIO-injected animals (2,261 \pm 623 vs. $282 \pm 101 \mu \mathrm{m}^{3} \times 10^{-6}$; $P=0.035$; Figure 3E). Furthermore, histological evaluation of LIBSinjected CM animals revealed the presence of MPIO bound to aggregated endovascular platelets (see below). These data confirm that there is significant binding of LIBS-MPIO to areas of CM pathology and demonstrate that the use of this targeted contrast agent enables detection of pathology before the onset of overt clinical signs.

In vivo MRI for platelet detection after TNF and IL-1 injection. To further investigate the mechanisms underlying cerebrovascular platelet aggregation in CM, we used MRI in conjunction with the LIBS-MPIO contrast agent to examine the spatial distribution of platelet aggregation in vivo following stereotactic injection of either TNF or IL-1 $\beta$ into the brains of normal mice.

In the TNF-injected animals using the LIBS-MPIO contrast agent, negative MRI contrast was observed bilaterally throughout the anterior portion of the forebrain (Figure 4A). Conversely, animals injected intracerebrally with IL-1 $\beta$ (Figure $4 \mathrm{~B}$ ) or saline (Figure 4C) showed no areas of MPIO binding (negative contrast) following injection of LIBS-MPIO. Nonspecific binding of LIBS-
MPIO was excluded using control-MPIO in TNF-injected mice (Figure 4D). As with mice injected with IL-1 $\beta$ or saline, these animals showed no alteration in signal intensity.

Using a 3D reconstruction of the original MRI data stack, we found binding of LIBS-MPIO to be enhanced in both cortical and striatal regions of the brain following TNF injection into the brain parenchyma (Figure 5A and Supplemental Movie 3), while no specific binding was evident following control-MPIO injection (Figure 5B and Supplemental Movie 4). As expected, at $12 \mathrm{~h}$ after TNF injection a similar binding pattern was found in both hemispheres, indicating bilateral aggregation, with slightly enhanced signal intensity changes on the side of injection. Volumetric quantification confirmed significantly greater LIBS-MPIO binding in TNF-injected animals compared with both IL-1 $\beta$ - and saline-injected animals, as well as TNF-injected animals receiving the control-MPIO agent (Figure 5C). These in vivo data indicate a role for TNF in platelet aggregation in cerebral vessels.

By immunohistochemistry, MPIO binding was apparent in TNF-injected animals receiving the LIBS-MPIO (Figure 6). On cresyl-violet-stained paraffin-embedded sections, binding of MPIOs to areas near the vascular wall was confirmed (Figure 6A). Furthermore, costaining with CD41 (GPIIb subunit) confirmed that binding of LIBS-MPIO was specific to areas of wall-adherent platelets (Figure 6B). Attached to the red-stained thrombus area, 2 MPIOs in different focus (owing to their location in different focal planes through the section) were evident. 
A

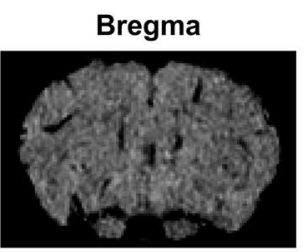

B

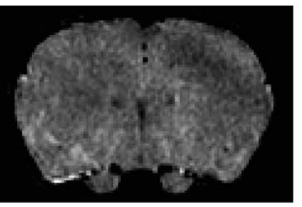

C

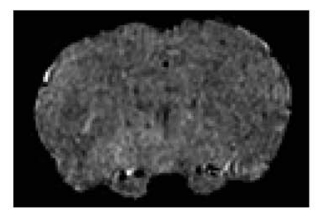

D

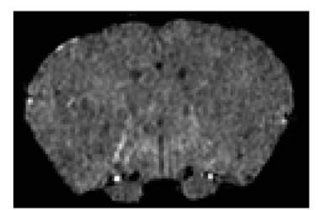

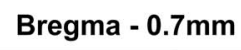
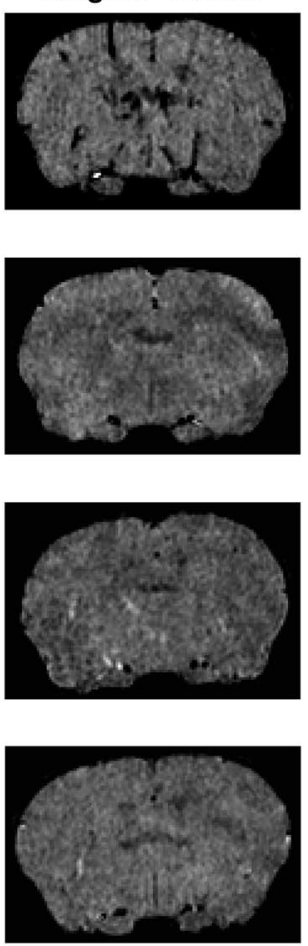
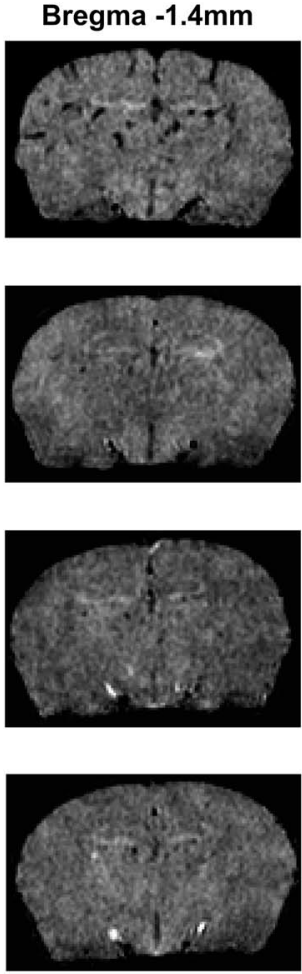

Bregma -2.1mm
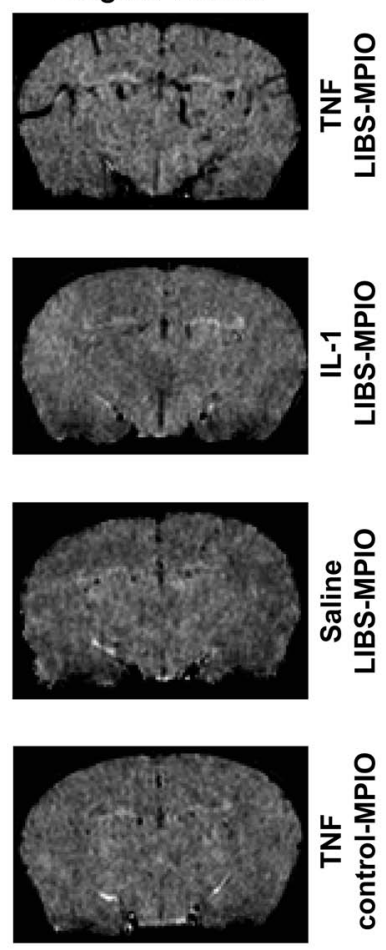

$\frac{\frac{\frac{0}{2}}{2}}{\frac{1}{\frac{1}{0}}}$

\section{Figure 4}

In vivo $\mathrm{T}_{2}$-weighted coronal images (4 images per brain, beginning at Bregma and moving backward in 700 - $\mu \mathrm{m}$ increments) from 3D gradientecho data sets each with approximately $90-\mu \mathrm{m}$ isotropic resolution. (A) Animal injected intrastriatally with $1 \mu \mathrm{g}$ TNF in $0.5 \mu \mathrm{l}$ saline $11.5 \mathrm{~h}$ prior to intravenous injection of LIBS-MPIO $(\sim 4.5 \mathrm{mg} / \mathrm{kg} \mathrm{Fe})$. Intense low-signal areas (black) reflect the specific retention of MPIO on activated platelets adhering to the cerebrovascular endothelium. (B and $\mathbf{C}$ ) In contrast, no effects of the LIBS-MPIO agent were detected in animals injected $11.5 \mathrm{~h}$ previously with $1 \mu \mathrm{g} \mathrm{IL}-1 \beta$ in $0.5 \mu \mathrm{l}$ saline (B) or with $0.5 \mu \mathrm{l}$ saline alone (C). (D) Similarly, no nonspecific effects of the control-MPIO contrast agent were detected in animals injected intrastriatally with $1 \mu \mathrm{g} \mathrm{TNF}$ in $0.5 \mu \mathrm{l}$ saline.

The number of platelets bound to the brain endothelium after the microinjection of TNF was highest at $12 \mathrm{~h}$ both in rats and in mice. To examine the relationship between the hypointensity volume and the number of platelets adherent in the vasculature, we compared the volume of MRI-detectable hypointensities with the number of adherent platelets determined immunohistochemically at 6,12 , and $24 \mathrm{~h}$ after microinjection of TNF in mice. Binding of the contrast agent to the microvasculature was maximal at $12 \mathrm{~h}$ as detected by MRI (Figure 6, D and E), and there was a correlation between the number of platelets present in the brain vasculature and the MPIO-dependent signal loss $\left(r^{2}=0.9503\right)$. This suggests that increased platelet load over time is directly related to increased LIBS-MPIO-induced signal reduction. In an animal injected with LIBS-MPIO $24 \mathrm{~h}$ after intracerebral TNF injection and imaged at both 2 and $10 \mathrm{~h}$ after LIBS-MPIO injection, the initial signal from the LIBS-MPIO present on the first scan was almost entirely absent in the later scan (Supplemental Figure 2). This finding suggests degradation of the contrast agent over the intervening period.

Cytokines and platelet aggregation. To establish whether the early expression of cytokines within the brain parenchyma is responsible for platelet adhesion, TNF, LT- $\alpha$, or IL- $1 \beta$ was injected directly into the brain parenchyma. These cytokines are all known to be expressed within the brain parenchyma in CM, but to our knowledge their role in platelet adherence was hitherto unknown. GPIIbpositive platelets became adherent to the luminal portion of the vasculature from $2 \mathrm{~h}$ after microinjection of TNF into the rat brain parenchyma (Figure 7, A and B). The number of platelet-positive elements peaked at $12 \mathrm{~h}$, but was still significantly increased $24 \mathrm{~h}$ after injection of TNF. This effect was also observed in mouse brain parenchyma, where platelet adhesion at the 12 -h time point reached the maximum compared with the 6- or 24-h time points (Figure $6 \mathrm{D}$ ). In contrast, injection of IL-1 $\beta$, LT- $\alpha$, or vehicle did not increase the number of platelet-positive elements (Figure 7B). Thus it appears that platelet binding to the brain endothelium is cytokine specific and that TNF is likely the principal mediator of endothelial platelet binding in CM. Interestingly, leukocytes, identified by the leukocyte common antigen, were recruited to the brain parenchyma or became adherent to the luminal portion of the vasculature over the 24-h period following injection of TNF into the striatum, but after the appearance of platelets in the microvasculature (Figure 7C). The recruited leukocytes were principally ED-1-positive cells, and no neutrophils were observed (data not shown). The recruited ED-1-positive cells were distinguished as 2 separate populations: parenchymal ED-1-positive cells, which had diapedesed into the parenchyma (Figure 7D), and vessel-associated ED-1-positive cells, which were associated with the luminal portion of the brain vasculature and appeared to be unable to diapedese into the parenchyma (Figure 7E). The number of ED-1-positive cells detected in the brain parenchyma increased from $12 \mathrm{~h}$ after injection $(P<0.05)$ and peaked at $24 \mathrm{~h}$ 
A
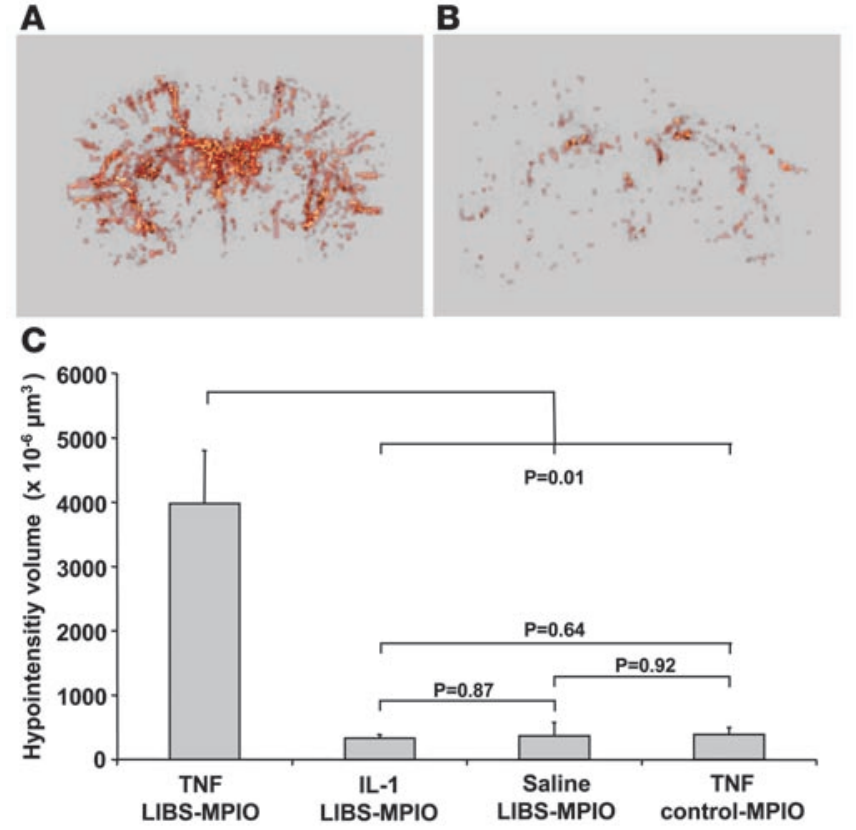

$(P<0.05)$ compared with vehicle-injected controls. A larger number of vessel-associated ED-1-positive cells was observed after $6 \mathrm{~h}$ $(P<0.05)$, again increasing over time to peak at $24 \mathrm{~h}(P<0.05)$, compared with vehicle-injected controls. Representative histology demonstrated the lack of cellular recruitment in the meninges (Figure 7F) and parenchyma (Figure 7G) of vehicle-injected controls and the presence of ED-1-positive cells in the parenchyma after TNF microinjection into the striatum (Figure $7 \mathrm{H}$ ). The ED-1-positive cells adherent to the vessel lumen were visualized using double-labeling immunohistochemistry (Figure 7I): vessels were located using an antibody to GLUT-1, a marker whose expression has been established on both the luminal and abluminal portions of the brain vasculature (22), together with an antibody against ED-1. These findings were confirmed using immunofluorescence and laser-scanning confocal microscopy (Figure 7J). Interestingly, the GLUT-1 confocal microscopy revealed that the platelets were binding to intact endothelium, which was unexpected.

In the meninges, recruited ED-1-positive mononuclear cells were observed as early as $2 \mathrm{~h}$ after intrastriatal TNF injection and increased dramatically over the 24 -h period (data not shown). The movement of ED-1-positive mononuclear cells across the menin-

\section{Figure 6}

Histology of TNF-injected brains with LIBS-MPIO injection. (A) Cresylviolet stain revealed binding of MPIO to areas on the vascular wall (arrows). (B) Binding of LIBS-MPIO to platelets or platelet thrombi was confirmed using immunohistochemistry for platelet-specific CD41: 2 MPIOs appearing in different focus and therefore of different shape were recognized at areas of platelet aggregation (arrows). (C) Similarly, MPIOs were detected on platelets and platelet thrombi in animals with $\mathrm{CM}$ (inset, high-power view of the region indicated by arrow). (D) Platelet-positive elements per injected hemisphere at 6, 12, and $24 \mathrm{~h}$ after intracerebral injection of TNF (diamonds) or saline (squares) in mice. (E) Quantification of the LIBS-MPIO-induced signal void in animals 6,12 , and $24 \mathrm{~h}$ after intracerebral TNF injection. Note the correlation between the number of platelet-positive elements over time and the LIBS-MPIO-induced signal void $\left(r^{2}=0.9503\right)$.

\section{Figure 5}

3D data and MRI signal quantification of animals with intracerebral TNF injection. (A) LIBS-MPIO binding pattern after intracerebral TNFinjection in a 3D reconstruction, showing the enhancement of cortical and central LIBS-MPIO binding. (B) Minimal background binding was observed in animals with control-MPIO injection. (C) Quantitative analysis showed significantly higher binding of LIBS-MPIO to TNF-injected brain areas compared with all controls.

geal vasculature appeared unrestricted, as large numbers of ED-1positive cells were observed abluminally (Figure $7 \mathrm{~K}$ ). These findings were supported by immunofluorescence and confocal microscopy (Figure 7L). Although not observed in the brain parenchyma, neutrophils were found in the meninges. These findings were also true of the choroid plexus, where a similar acute inflammatory response was observed after $2 \mathrm{~h}$ and increased to $24 \mathrm{~h}$ (data not shown).

\section{Discussion}

We report the application of a functional activation-specific platelet contrast agent for the detection of vascular platelet adhesion in vivo. The use of this targeted contrast agent, which we believe to be novel, has contributed significantly to our understanding of the mechanism of platelet activation in the pathology of CM. The findings in this study are multiple: (a) Using the activation-specific LIBS-MPIO contrast agent, diagnosis of the life-threatening pathology of CM involving platelet aggregation was possible at an earlier stage than either clinical findings or conventional MRI allow. The functional approach used here enables imaging of activated platelets and platelet thrombi that cannot be detected by routine MRI sequences. (b) Platelet binding to the brain endothelium was cytokine specific, and TNF is likely to be the principal mediator in CM. (c) After the appearance of platelets in the microvasculature, TNF, but not IL-1 $\beta$, induced the adherence of mononuclear cells to the cerebral vasculature in a manner very reminiscent of CM pathology. (d) These results

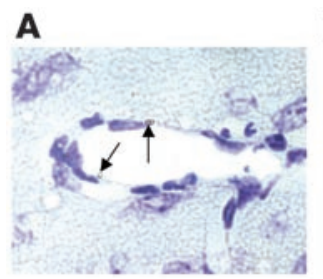

B

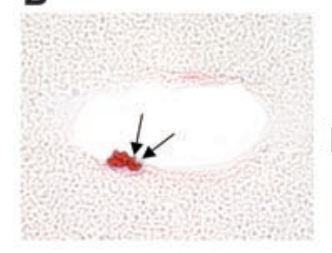

C

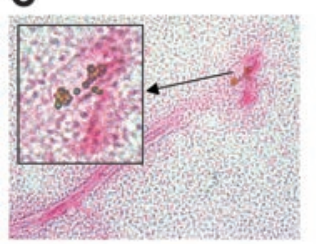

D

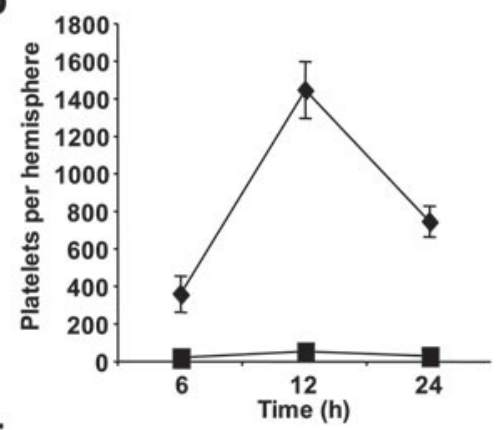

E

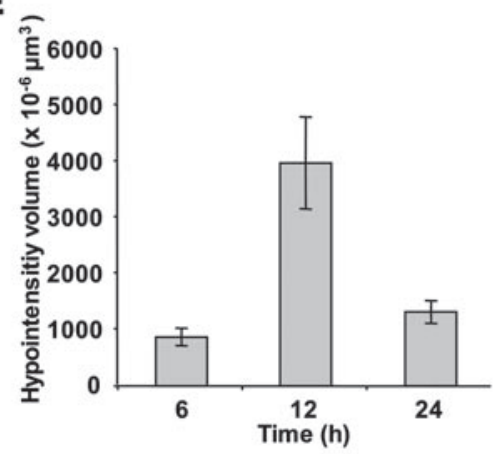


A

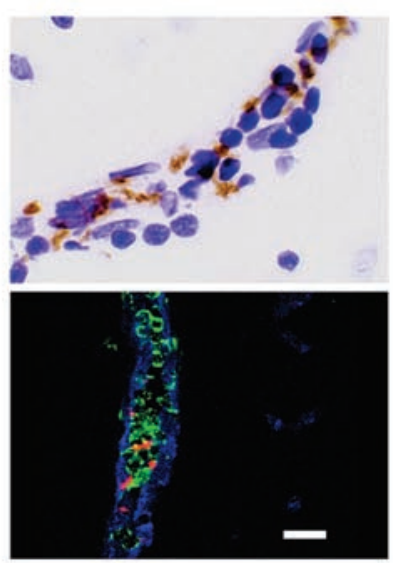

C
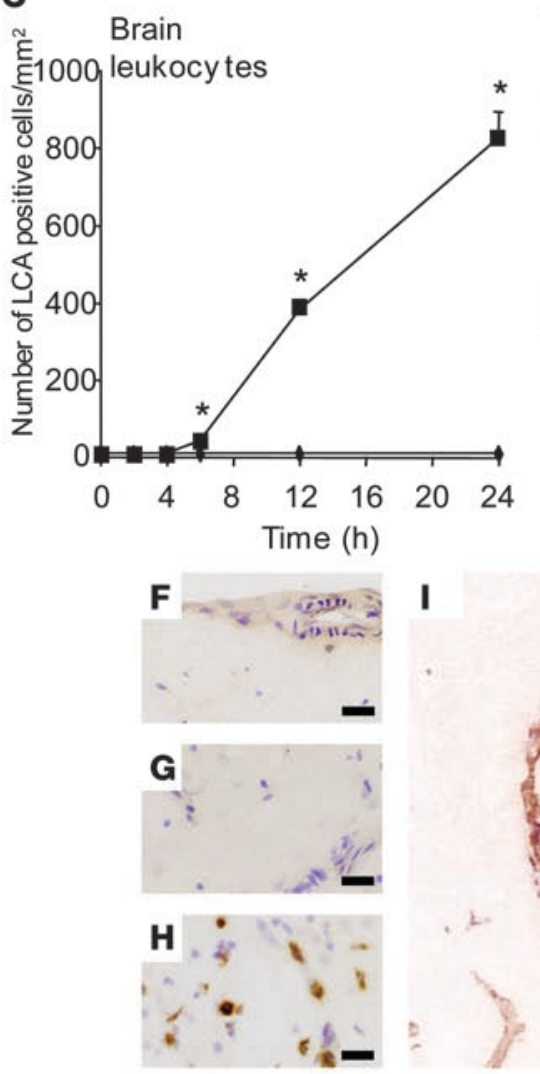
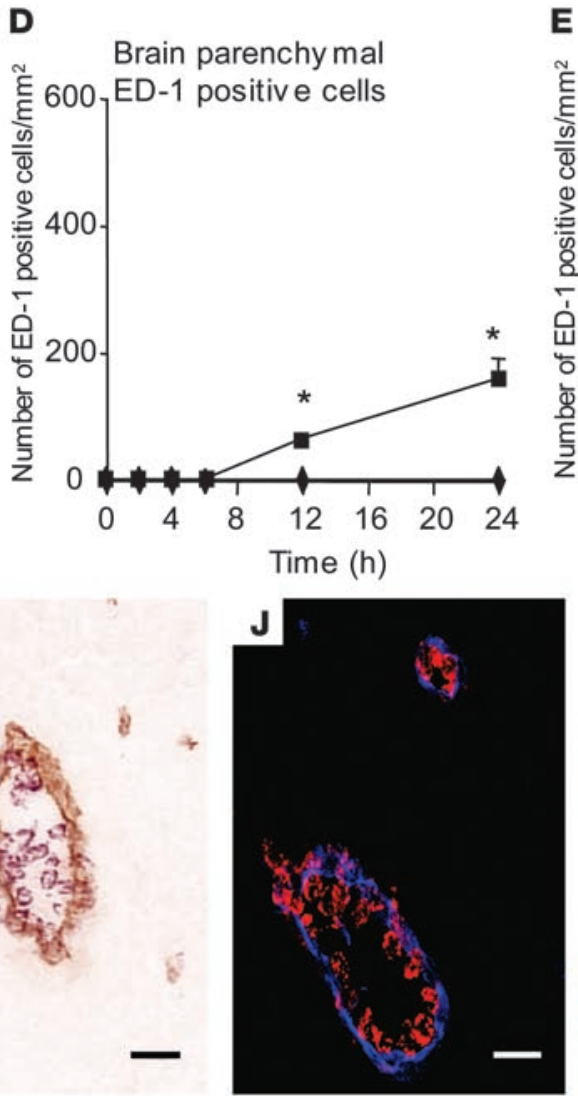

$\mathbf{E}$
B
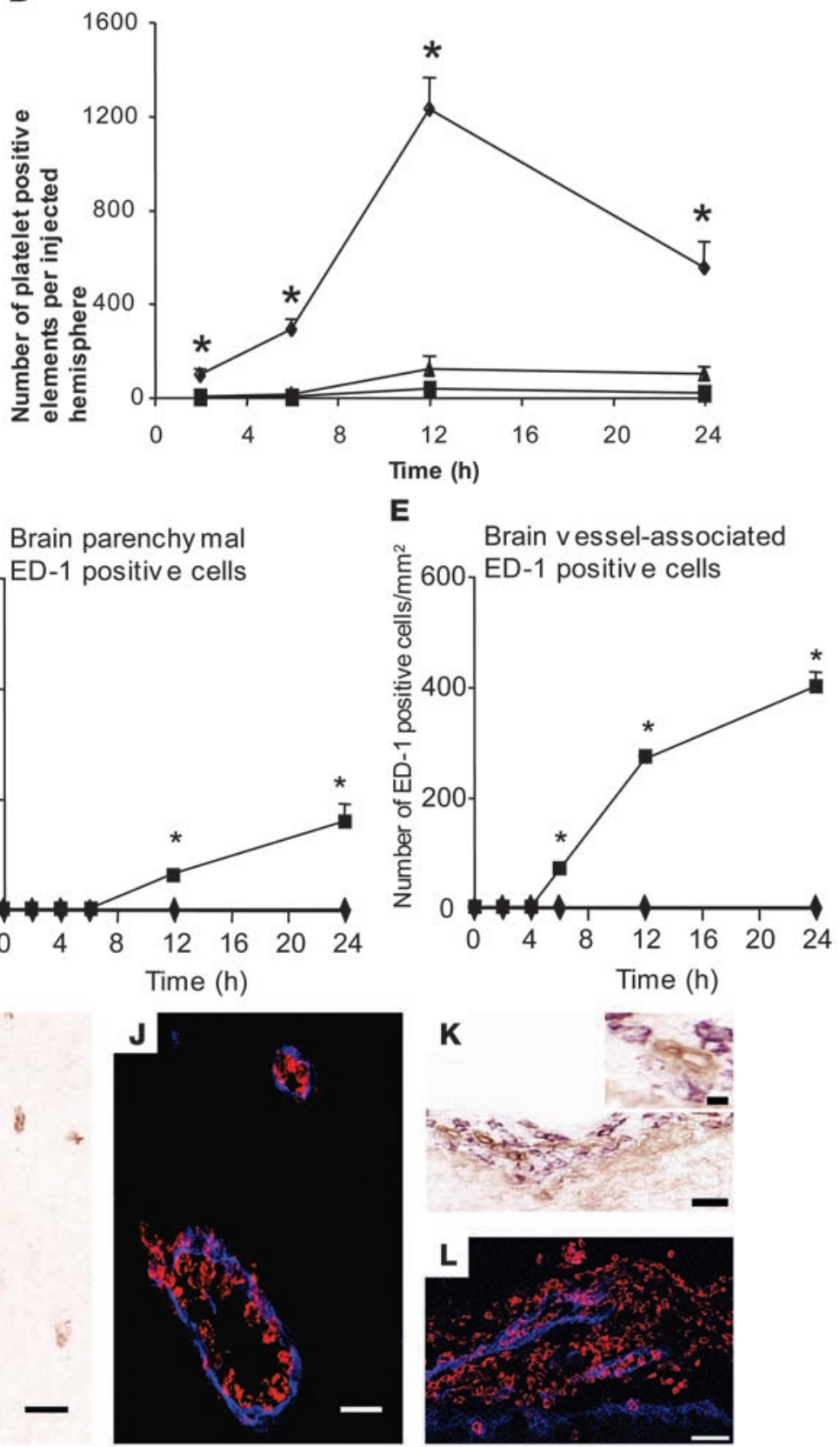

\section{Figure 7}

Platelet and leukocyte accumulation in the brain following the intrastriatal injection of TNF. (A) Top: Platelets localized within blood vessel in the brain close to the injection site (brown, DAB), visualized by cresyl-violet counterstain. Bottom: Confocal triple-labeling immunocytochemistry identified platelets (red) and ED-1-positive cells (green) inside a vessel with an intact endothelium, as revealed by GLUT1 (blue) staining. (B) Number of GPIIb/lla-positive elements in the injected hemisphere following microinjection into the striatum of $1 \mu \mathrm{g}$ TNF (diamonds), $1 \mathrm{ng} \mathrm{IL-1}$ (triangles), or saline (squares) in a volume of $1 \mu \mathrm{l}$ for rats or $0.5 \mu \mathrm{l}$ for mice. (C-E) Total brain leukocytes, as identified by leukocyte common antigen (LCA; C), ED-1-positive recruited monocytes and activated microglial cells present in the brain parenchyma (D), and ED-1-positive recruited monocytes associated with the luminal portion of the brain vasculature (E), detected by immunohistochemistry over $24 \mathrm{~h}$ following intrastriatal injection of TNF (squares) or saline vehicle (diamonds). (F-H) Representative photographs showing the absence of leukocytes in the meninges (F) or parenchyma (G) and their presence in the parenchyma following injection of TNF $(\mathbf{H})$ after immunohistochemical labeling for leukocyte common antigen. (I-L) Double-labeling immunocytochemistry ( $\mathbf{I}$ and $\mathbf{K}$ ) and confocal imaging ( $\mathbf{~}$ and $\mathbf{L})$ of ED-1-positive cells and the brain vasculature (as identified by GLUT-1) highlight the differences in mononuclear cell recruitment patterns in the parenchyma (I and $\mathbf{J})$ and meninges (K and $\mathbf{L})$. Note in the meninges the presence of large numbers of ED-1-positive cells (blue, VIP; red, FITC) that appear to have free passage from the vasculature (brown, $\mathrm{DAB}$; blue, AF-636) compared with the same cells in the parenchyma that appear vessel associated. (K, inset) High-power view of extravascular ED-1-positive cells. ELISA results are expressed as pg MCP-1/mg total protein \pm SEM. Cell numbers in brain are expressed per $\mathrm{mm}^{2} \pm \mathrm{SEM}$. ${ }^{*} P<0.05$ versus vehicle control. Scale bars: $20 \mu \mathrm{m}$ (A and $\left.\mathbf{F}-\mathbf{J}\right) ; 40 \mu \mathrm{m}(\mathbf{K}$ and $\mathbf{L}) ; 10 \mu \mathrm{m}(\mathbf{K}$, inset). 
demonstrate that the molecular imaging strategy applying LIBSMPIO may be used for the detection of pathologies involving platelets, constituting a noninvasive in vivo examination with high sensitivity and specificity.

Imaging cerebrovascular platelet aggregation with LIBS-MPIO. Conjugation of superparamagnetic particles to antibodies or peptidomimetic peptides has previously been used to selectively image cellular receptors expressed in various disease conditions $(11,23$, 24). Gadolinium-based contrast agents shorten the $T_{1}$ relaxation time of protons and provide positive contrast on $\mathrm{T}_{1}$-weighted images (25). Sipkins et al. have previously imaged ICAM-1 expression in rat brain ex vivo using paramagnetic liposomes (26), and we have targeted E-selectin expression in rat brain in vivo using a Sialyl Lewis ${ }^{x}$ mimetic moiety conjugated to Gd-DTPA (9). The major limitation of gadolinium-based agents is the small quantity of gadolinium that can be delivered to an endothelial monolayer, which limits the resulting contrast effects. Shapiro et al. recently described the use of MPIOs for cellular imaging and tracking, which provide substantially greater contrast than do gadoliniumbased agents (14). The detection of MPIOs in single cells in animal-based in vivo models has been described by the same group recently (27). Therefore, the use of micron-sized particles for the detection of sparse and difficult-to-access functional epitopes was highly attractive, and the excellent contrast properties improved the feasibility of this approach. We have recently described a similar method for detecting VCAM-1 expression on the vascular endothelium in vivo early in CNS inflammation, using an anti-VCAM-1 antibody conjugated to MPIO (15).

An important advantage of the current study is the use of a single-chain antibody in the LIBS-MPIO contrast agent, thus enabling binding to function-specific targets such as LIBS on activated GPIIb/IIIa. Single-chain antibodies are also less immunogenic than IgG-sized proteins because of the lack of the Fc-regions and, in spite of small protein size, allow the attachment of micron-sized iron oxide particles to target epitopes, as shown in the present study.

Differential binding patterns of the LIBS-MPIO contrast agent were evident in each experimental model. In TNF-injected animals, agent binding was observed in both hemispheres encompassing both cortical and striatal regions, although more contrast was evident in the injected (left) hemisphere. We have previously shown widespread (cortical and striatal) effects of TNF following a single intrastriatal bolus injection into rat brain (28). Immunohistochemically, adhesion molecule expression and platelet adhesion was found to be bilateral at the time point used in the current study, although - in accord with the MRI findings - more staining was present in the injected hemisphere. Conversely, in CM animals cortical binding was more pronounced than subcortical contrast changes. Maximum binding of LIBS-MPIO to platelets in the microvasculature was highest at $12 \mathrm{~h}$, as detected by MRI, correlating to the number of platelets present $\left(r^{2}=0.9503\right)$. This suggests that increased platelet load over time is directly reflected by increased LIBS-MPIO-induced signal void.

The concept of functional GPIIb/IIIa targeting opens up the possibility of detecting and imaging in vivo platelet aggregation, which has only been observed in postmortem tissue from the brains of patients with CM, multiple sclerosis, HIV-dementia, and bacterial meningitis. We have previously described the application of this contrast agent for the detection of activated platelets in a mouse model of platelet aggregation on mechanically injured endothelium using ex vivo MRI (19), and the potential applications of this or similar contrast agents are far reaching. In particular, the use of such an agent for monitoring antiplatelet therapy may have considerable utility across a number of diseases, including stroke, thrombosis, and cardiovascular disease, as well as in CM as described here.

Another important property for purposes of therapy monitoring is the biodistribution of the particles as well as the duration of contrast agent binding to the targeted receptors. In animals imaged for the first time $24 \mathrm{~h}$ after intracerebral TNF injection, the signal from the LIBS-MPIO in the brain detected $2 \mathrm{~h}$ after agent injection was almost entirely lost $10 \mathrm{~h}$ later. This suggests that the contrast agent is degraded and is not sequestered in the brain, which is an important prerequisite for serial imaging. Concerning the biodistribution of MPIO after injection, we have recently shown that MPIOs were identified in lung, kidney, liver, and spleen after 30 min with redistribution to the spleen and, to a lesser extent, the liver after $24 \mathrm{~h}$ (29). Importantly, there was no evidence of infarction, hemorrhage, edema, inflammation, or MPIO extravasation in any of these organs at either time point. The liver and spleen signals decayed over a week-long period, but the route of excretion is unclear (data not shown).

Cytokines and platelet aggregation. A broad spectrum of proinflammatory agents rapidly upregulates adhesion molecules on cerebral endothelium, in particular ICAM-1 and the selectins, in a time course comparable to that demonstrated on non-CNS endothelium. Notably, leukocyte recruitment is often negligible (30), and little is known about adhesion of platelets in CM models. IL-1 $\beta$ and TNF both upregulate ICAM-1 $(31,32)$ and selectins $(33,34)$ on the brain endothelium, and therefore, the differential binding of platelets to the brain endothelium following the microinjection of these cytokines into the brain was surprising. However, non-adhesion molecule-related vascular events, such as cytokine-induced volume changes, may play a role. We have previously shown that following intrastriatal injection of TNF there is a decrease in regional cerebral blood volume (rCBV) (28), which is in contrast to the increased rCBV induced by IL-1 $\beta$ (35). TNF-induced reduction in $\mathrm{rCBV}$ may promote the adhesion of platelets and subsequent recruitment of monocytes. It is of interest to note that monocyte recruitment follows platelet adhesion and suggests that the adhesion of platelets provides a scaffold for the subsequent recruitment of monocytes, which happens at a time when regional blood flow has returned to normal (28).

Limitations of LIBS-MPIO. In this work, commercially available MPIOs were used. The polystyrene coating of these specific particles may not be suitable for use in humans owing to their potential toxicity, although no adverse events were observed in any of the mice injected with MPIO-based contrast agents. Due to the histidine-tag conjugation technique, the bond between antibody and MPIO remained stable for approximately $4 \mathrm{~h}$; after this time, a significant reduction in the efficacy of the agent was observed (data not shown). Research on more stable preparations is ongoing.

Conclusion. This study provides proof of concept that in experimental models of human neurological diseases, targeted contrast agents can be used to detect pathology earlier than conventional, clinically used MRI approaches or clinical assessment. Wall-adherent platelets were detected noninvasively in vivo in a model of CM, prior to the onset of clinical symptoms, using a functional MRI contrast agent that targeted LIBS on activated GPIIb/IIIa receptors. In contrast, conventional MRI techniques failed to reveal the presence of CNS pathology before the appearance of overt clinical signs. Owing to the high specificity and sensitivity of our contrast 
agent, we were able to identify TNF as the mediator responsible for platelet aggregation. Furthermore, we were able to demonstrate that due to disappearance of the contrast agent signal over time, serial imaging seems possible. These results demonstrate the potential of such agents in enabling the noninvasive assessment of pathological mechanisms in disease models for diagnostic purposes, mechanistic studies, and monitoring of therapy.

\section{Methods}

Single-chain antibody generation, conjugation to MPIOs, and in vitro binding studies. The mAb anti-LIBS 145 binds to GPIIb/IIIa only in its active conformation and demonstrates strong binding to ADP-activated platelets in the presence of fibrinogen. Generation of anti-LIBS 145 has been previously described in detail (36). In brief, the $\mathrm{mAb}$ anti-LIBS 145-expressing hybridoma cell line was used as the basis for the cloning of an anti-LIBS single-chain antibody, $\mathrm{scFv}$. mRNA of this hybridoma cell line was prepared and reverse transcribed using an oligo-dT primer. The variable regions of the antibody's heavy and light chain were amplified by PCR and cloned into the PHOG21 vector, TG1 E. coli. Individual clones were assessed for LIBS-typical binding to GPIIb/IIIa in flow cytometry using activated platelets. Finally, the best binding $s c \mathrm{Fv}_{\text {LIBS }}$ was produced in LB media containing ampicillin and glucose. Centrifuged and pelleted bacteria were resuspended in BugBuster (Novagen) and again centrifuged, and the supernatant containing soluble protein was kept on ice after adding complete protease inhibitor (Roche). The supernatant was mixed with $\mathrm{Ni}^{2+}$-Agarose (Qiagen) binding His6-tagged proteins. Finally, the $\mathrm{scFv}$ was eluted at high imidazole concentrations and dialyzed to PBS. Functionality of the $\mathrm{scFv}$ preparations was evaluated by flow cytometry.

For the irrelevant control antibody, exchange of the arginine in the RXD motif of the heavy-chain CDR3 region of a platelet single-chain antibody was performed to achieve a nonfunctional antibody for control purposes. The generation and purification of this antibody was performed in the same way as described above.

For construction of the contrast agent, autofluorescent cobalt-functionalized MPIOs $(1 \mu \mathrm{m})$ were conjugated to the histidine-tag of the LIBS/control single-chain antibody according to the manufacturer's protocol (Dynal Biotech). In brief, $1 \mathrm{mg}$ of beads was incubated with the LIBS/control antibody for $10 \mathrm{~min}$ at room temperature to bind approximately $10 \mathrm{mg}$ of histidinetagged antibody. The tube containing the suspension was then placed on a magnet until the beads had migrated to the side of the tube and the supernatant was discarded. This washing step was repeated 4 times using a binding and washing buffer as described by the manufacturer (Dynal Biotech). MPIOs conjugated to the LIBS antibody are referred to herein as LIBS-MPIO, MPIOs conjugated to control antibodies are referred to as control-MPIO.

To examine the binding characteristics of the LIBS-MPIO contrast agent, blood from healthy human volunteers was obtained. Studies in humans were reviewed and approved by the Alfred Human Research Ethics Committee (Melbourne, Victoria, Australia). Informed consent was obtained from all study participants. After the centrifugation of whole blood $(228 \mathrm{~g}$ for $10 \mathrm{~min}$ ), $50 \mu \mathrm{l}$ of platelet-rich plasma was incubated with either $20 \mu \mathrm{M}$ ADP (a potent platelet activator) or vehicle on a microscope slide. LIBSMPIO, control-MPIO, or nonfunctionalized MPIOs were applied and incubated under continuous and careful rotation. After $10 \mathrm{~min}$ the slides were washed and coverslipped, and MPIO binding was evaluated.

Murine malaria model. Female C57BL6 mice were purchased from Charles River. Infections were initiated by i.p. injection of $10^{6} P$. berghei ANKA-parasitized rbcs per mouse. Parasitemia and health status were monitored on a daily basis in accordance with our UK Home Office license. All animal experiments were reviewed and approved by the UK Home Office (London, United Kingdom). The level of parasitemia was evaluated on blood smears after Giemsa staining.
Stereotaxic microinjection of recombinant cytokines. The malaria model is mouse specific, but the histological outcomes following microinjection of cytokines in the rat or mouse brain are conserved $(15,29,37,38)$. Thus we used rats or mice, depending on the availability of complementary antibodies, to examine the profile of platelet binding and microvessel integrity after the microinjection of cytokine into the brain.

Rat-recombinant TNF and IL- $1 \beta$ were obtained from the National Institute for Biological Standards and Controls (NIBSC). Rat-recombinant LT- $\alpha$ and mouse-recombinant TNF were purchased from R\&D Systems. The cytokines were dissolved in endotoxin-free saline (vehicle). The cytokines contained a maximum of $100 \mathrm{IU}$ endotoxin/mg cytokine (corresponding to 10 parts per million by weight), which, in view of previous studies (39), was considered negligible in the context of these experiments.

We used 12-week-old male Wistar rats or 8-week-old NMRI mice for the injection of the recombinant cytokines (Charles River). Both rats and mice responded to the injection of cytokines in an identical manner. In each experiment, at least 3 animals were used per group. All surgical procedures were performed under an operating microscope (Wild M650; Leica). Stereotaxic surgery was performed as described previously (40). Briefly, anesthetized rats were held in a stereotaxic frame. A small hole was drilled in the skull, and $1 \mu \mathrm{g}$ of TNF or LT- $\alpha, 1 \mathrm{ng}$ of IL- $1 \beta$, or saline in a volume of $1 \mu \mathrm{l}$ for rats or $0.5 \mu \mathrm{l}$ for mice was microinjected into the striatum (an area of brain parenchyma containing both gray and white matter) with a glass capillary needle (tip $<50 \mu \mathrm{m}$ ).

Tissue collection. After appropriate survival times, animals were deeply anesthetized with sodium pentobarbitone. Transcardiac perfusions were carried out using heparinized saline. Tissue was removed and either frozen in liquid nitrogen or embedded in Tissue Tek and frozen for histology.

Identification of leukocytes and platelets. Frozen 10- $\mu \mathrm{m}$-thick serial coronal sections were cut from tissue blocks. Using immunohistochemistry, neutrophils were identified using the anti-neutrophil serum HB199 (41), activated microglia cells and recruited monocytes were identified using the ED-1 and antibody recognizing a lysosomal membrane marker on myeloid cells (Serotec), and total recruited leukocytes were identified using leukocyte common antigen marker with a combination of the antibodies OX1 and OX30 (Serotec and Cedarlane Laboratories Ltd.). In the brain, ED-1-positive cells were subdivided into parenchymal and vessel-associated cells. Parenchymal cells were defined as those that were present on the abluminal surface of the vessel and within the parenchyma; vessel-associated cells were defined as those cells adherent to the luminal surface of the vasculature. The numbers of positive cells present in the brain were quantitated. For each tissue section, 4 representative fields were chosen, and the average number of positive cells was calculated and expressed as number of cells per $\mathrm{mm}^{2}$.

The p55 mouse anti-rat platelet GPIIa monoclonal antibody was a kind gift from Kirin Brewery Co. Ltd. The numbers of platelet-positive elements present in the brain were quantitated. For each tissue section, 6 representative fields were chosen, and the average number of positive elements was calculated and expressed as number of discrete elements per $\mathrm{mm}^{2}$.

For the platelet detection in mice used for in vivo MRI, mouse platelets were detected using rat anti-mouse glycoprotein IIb (CD41) polyclonal antibody (Clone MWReg30; GeneTex) in a dilution of 1:25 overnight at $4{ }^{\circ} \mathrm{C}$. Primary antibody was detected using a rabbit anti-rat biotinylated secondary antibody (Vectastain ABC-AP Kit; Vector Laboratories) and an alkaline phosphate reaction (Alkaline Phosphatase Substrate Kit II; Vector Laboratories).

Cresyl-violet-stained brain sections were examined for the presence of MPIO. Digital light microscopy images of histological sections were captured with a Cool Snap Pro color video camera (Media Cybernetics) mounted on a light microscope (Leica DM R).

Double- and triple-labeling immunobistochemistry and immunofluorescence. Frozen $10-\mu \mathrm{m}$ coronal sections were fixed in ethanol. A rabbit polyclonal antibody to GLUT-1 was used to identify the vessel surfaces of the brain. 
The glucose transporter has previously been established as present on both the luminal and abluminal sides of all vessels within the brain (22). GLUT-1 antibody was a gift from I. Simpson (Penn State College of Medicine, Hershey, Pennsylvania, USA). GLUT-1 was detected using standard $\mathrm{ABC}$ procedures as described above and revealed with diaminobenzidine (DAB; brown precipitate). ED-1 was subsequently identified using immunohistochemistry and revealed with VIP (blue precipitate; Vector Laboratories) as described above. For double-labeling immunofluorescence, GLUT-1 was revealed with chicken anti-rabbit Alexa Fluor 636 (Invitrogen), and ED-1 was identified with an anti-ED-1 directly labeled to FITC (Serotec), according to the manufacturer's instructions. Sections were analyzed by laser scanning confocal microscopy. Double-stained images presented are all 3D reconstructions where AF-636 is presented as blue labeling and FITC as red labeling. Triple-stained images presented are all 3D reconstructions where AF-636 (GLUT-1) is presented as blue labeling, FITC (ED-1) is shown as green labeling, and RITC (platelets) is shown as red labeling.

In vivo MRI of CM mice. MRI data were acquired using a 7-Tesla horizontal bore magnet with a Varian Inova spectrometer (Varian). Animals $(n=3$ per group) were imaged at days 6 and 7 after inoculation. One additional animal was imaged at day 5 . Animals were anesthetized with $0.5 \%-1.5 \%$ isoflurane in $70 \% \mathrm{~N}_{2} \mathrm{O}$ and $30 \% \mathrm{O}_{2}$ and positioned in an Alderman-Grant resonator. Heart rate, as monitored by ECG, was maintained at approximately 500-550 bpm in all animals, and body temperature was maintained at $37^{\circ} \mathrm{C}$ with a MRI-compatible homeothermic blanket and probe. $\mathrm{T}_{2}$ maps (echo time [TE], 0.02, 0.04, and $0.06 \mathrm{~s}$ ) were acquired, and regions of interest (hippocampus, cortex, and striatum) were selected on the slice at the same position as those depicted in Figure 1, A and C. $\mathrm{T}_{1}$-weighted images (repetition time [TR], $0.5 \mathrm{~s}$; TE, $0.02 \mathrm{~s}$ ) were acquired before and 10 min after Gd-DTPA injection to assess blood-brain barrier breakdown. A $\mathrm{T}_{2}$-weighted $3 \mathrm{D}$ gradient-echo data set was acquired (flip angle, $35^{\circ} ; \mathrm{TR}, 15 \mathrm{~ms}$; TE, $7 \mathrm{~ms}$; field of view, $22.5 \times 11.2 \times 31.6 \mathrm{~mm}$; matrix size, $192 \times 96 \times 360$; 6 averages; total acquisition time, about $30 \mathrm{~min}$ ). The midpoint of the acquisition was $1.8 \pm 0.4 \mathrm{~h}$ after LIBS-MPIO or control-MPIO injection ( $n=3$ per group). Data were zero-filled to $256 \times 128 \times 360$ and reconstructed offline, with a final isotropic resolution of $88 \mu \mathrm{m}^{3}$.

In vivo MRI of cytokine-injected mice. Mice were injected via a tail vein with the LIBS-MPIO or control-MPIO contrast agent $\left(4 \times 10^{8}\right.$ beads; $4.5 \mathrm{mg}$ $\mathrm{Fe} / \mathrm{kg}$ body weight; $n=3$ per group) $11.5 \pm 3.3 \mathrm{~h}$ after intracerebral injection of either saline vehicle or recombinant cytokine (TNF or IL-1 $\beta$ ). To examine the temporal relationship between the MRI MPIO signal and the number of platelets bound to the cerebral vasculature, we also injected, via a tail vein, the LIBS-MPIO contrast agent $5.1 \pm 0.1 \mathrm{~h}$ or $24.2 \pm 0.1 \mathrm{~h}$ after the intracerebral injection of TNF.

Furthermore, to establish whether serial imaging would be possible with the LIBS-MPIO contrast agent, in 1 animal injected with TNF $24 \mathrm{~h}$ prior to LIBS-MPIO injection we repeated the MRI measurements 10 hours after LIBS-MPIO contrast agent injection.

1. Marsh, K., and Snow, R.W. 1999. Malaria transmis sion and morbidity. Parassitologia. 41:241-246.

2. Wassmer, S.C., Combes, V., Candal, F.J., JuhanVague, I., and Grau, G.E. 2006. Platelets potentiate brain endothelial alterations induced by Plasmodium falciparum. Infect. Immun. 74:645-653.

3. Grau, G.E., et al. 1993. TNF-induced microvascular pathology: active role for platelets and importance of the LFA-1/ICAM-1 interaction. Eur. Cytokine Netw. 4:415-419.

4. Grau, G.E., and Lou, J. 1993. TNF in vascular pathology: the importance of platelet-endothelium interactions. Res. Immunol. 144:355-363.

5. Combes, V., et al. 2004. Pathogenic role of P-selectin in experimental cerebral malaria: importance of the endothelial compartment. Am. J. Pathol.
Following MPIO injection, animals were placed in an Alderman-Grant resonator and positioned in the magnet. During MRI, anesthesia was maintained with $1.7 \%-2.5 \%$ isoflurane in $70 \% \mathrm{~N}_{2} \mathrm{O}$ and $30 \% \mathrm{O}_{2}$, ECG was monitored throughout, and body temperature was maintained at $\sim 37^{\circ} \mathrm{C}$ with a circulating warm water system. $\mathrm{A} \mathrm{T}_{2}$-weighted $3 \mathrm{D}$ gradient-echo data set was acquired (flip angle, $35^{\circ}$; TR, $50 \mathrm{~ms}$; TE, $5 \mathrm{~ms}$; field of view, $22.5 \times 22.5 \times 31.6 \mathrm{~mm}$; matrix size, $192 \times 192 \times 360$; 2 averages; total acquisition time, about $1 \mathrm{~h}$ ). The midpoint of the acquisition was $1.8 \pm 0.2 \mathrm{~h}$ after MPIO injection. Data were zero-filled to $256 \times 256 \times 360$ and reconstructed offline, with a final isotropic resolution of $88 \mu \mathrm{m}^{3}$. All in vivo procedures were approved by the UK Home Office.

MRI data analysis. In each image of the MRI, the brain was masked manually to exclude extracerebral structures. Quantitative analysis was performed in 41 contiguous slices per brain, spanning a depth of $3.6 \mathrm{~mm}$ from the dorsal hippocampus ventrally. Areas of low signal were segmented. To control for minor variations in absolute signal intensity among individual scans, low-signal areas were calibrated on 10 evenly spaced slices per brain. The median signal intensity value was then applied to signal intensity histogram-based fully automated batch analysis of the entire 41-slice sequence. In this way, masks were generated corresponding to areas that were both within the brain and of defined low signal intensity. Voxel volumes were summed and expressed as raw volumes in $\mu \mathrm{m}^{3}$ with no surface rendering or smoothing effects. Segmentation and volumetric quantification was performed using ImagePro Plus software (version 4.5.1; Media Cybernetic) by an operator blinded to the origin of all data.

Statistics. Data were presented as mean \pm SEM at each time point. Where statistical analysis was used, data were analyzed by $t$ tests. Results were considered significant when $P<0.05$.

\section{Acknowledgments}

We thank Andrew Lowe, Nicole Bassler, and Irene Neudorfer for technical assistance and Tom Bannister for his help with the data quantification. This work was funded by grants from the Medical Research Council (to N.R. Sibson and D.C. Anthony), the Wellcome Trust (to R.P. Choudhury), and the NHMRC and National Heart Foundation of Australia (to K. Peter).

Received for publication July 19, 2007, and accepted in revised form December 19, 2007.

Address correspondence to: Daniel C. Anthony, Department of Pharmacology, University of Oxford, Mansfield Road, Oxford OX1 3QT, United Kingdom. Phone: 44-1865-281136; Fax: 44-1865271853; E-mail: daniel.anthony@pharm.ox.ac.uk.

Constantin von zur Muhlen, Nicola R. Sibson, Karlheinz Peter, and Sandra J. Campbell contributed equally to this work.
164:781-786

6. Grau, G.E., et al. 2003. Platelet accumulation in brain microvessels in fatal pediatric cerebral malaria. J. Infect. Dis. 187:461-466.

7. Lou, J., et al. 1997. Platelets play an important role in TNF-induced microvascular endothelial cell pathology. Am. J. Pathol. 151:1397-1405.

8. Grau, G.E., and Lou, J.N. 1995. Experimental cerebral malaria: possible new mechanisms in the TNF-induced microvascular pathology. Soz. Praven tivmed. 40:50-57.

9. Sibson, N.R., et al. 2004. MRI detection of early endothelial activation in brain inflammation. Magn. Reson. Med. 51:248-252.

10. Hunt, N.H., and Grau, G.E. 2003. Cytokines: accelerators and brakes in the pathogenesis of cerebral malaria. Trends Immunol. 24:491-499.

11. Spuentrup, E., et al. 2005. Molecular magnetic resonance imaging of coronary thrombosis and pulmonary emboli with a novel fibrin-targeted contrast agent. Circulation. 111:1377-1382.

12. Nahrendorf, M., et al. 2006. Noninvasive vascular cell adhesion molecule-1 imaging identifies inflammatory activation of cells in atherosclerosis. Circulation. 114:1504-1511.

13. Shapiro, E.M., Skrtic, S., and Koretsky, A.P. 2005. Sizing it up: cellular MRI using micron-sized iron oxide particles. Magn. Reson. Med. 53:329-338.

14. Shapiro, E.M., et al. 2004. MRI detection of single particles for cellular imaging. Proc. Natl. Acad. Sci.U.S. A. 101:10901-10906.

15. McAteer, M.A., et al. 2007. In vivo magnetic reso- 
nance imaging of acute brain inflammation using microparticles of iron oxide. Nat. Med. 13:1253-1258

16. Schwarz, M., et al. 2006. Conformation-specific blockade of the integrin GPIIb/IIIa: a novel antiplatelet strategy that selectively targets activated platelets. Circ. Res. 99:25-33.

17. Schwarz, M., et al. 2004. Single-chain antibodies for the conformation-specific blockade of activated platelet integrin alphaIIbbeta 3 designed by subtractive selection from naive human phage libraries. FASEB J. 18:1704-1706.

18. Stoll, P., et al. 2007. Targeting ligand-induced binding sites on GPIIb/IIIa via single-chain antibody allows effective anticoagulation without bleeding time prolongation. Arterioscler. Thromb. Vasc. Biol. 27:1206-1212.

19. von zur Mühlen, C., et al. 2007. Magnetic resonance imaging of platelets on wire-injured mouse femoral arteries using activation-specific anti-GP IIb/IIIa single chain antibodies conjugated to microparticles of iron oxide [abstract]. J. Am. Coll. Cardiol. 49(Suppl.):108A.

20. Sun, G., et al. 2003. Inhibition of platelet adherence to brain microvasculature protects against severe Plasmodium berghei malaria. Infect. Immun. 71:6553-6561.

21. van der Heyde, H.C., Nolan, J., Combes, V., Gramaglia, I., and Grau, G.E. 2006. A unified hypothesis for the genesis of cerebral malaria: sequestration, inflammation and hemostasis leading to microcirculatory dysfunction. Trends Parasitol. 22:503-508.

22. Guerin, C., et al. 1990. The glucose transporter and blood-brain barrier of human brain tumors. Ann. Neurol. 28:758-765.

23. Winter, P.M., et al. 2003. Improved molecular imaging contrast agent for detection of human thrombus. Magn. Reson. Med. 50:411-416.
24. Lipinski, M.J., et al. 2006. MRI to detect atherosclerosis with gadolinium-containing immunomicelles targeting the macrophage scavenger receptor. Magn. Reson. Med. 56:601-610.

25. Runge, V.M., et al. 1985. Initial clinical evaluation of gadolinium DTPA for contrast-enhanced magnetic resonance imaging. Magn. Reson. Imaging. 3:27-35.

26. Sipkins, D.A., et al. 2000. ICAM-1 expression in autoimmune encephalitis visualized using magnetic resonance imaging. J. Neuroimmunol. 104:1-9.

27. Shapiro, E.M., Sharer, K., Skrtic, S., and Koretsky, A.P. 2006. In vivo detection of single cells by MRI. Magn. Reson. Med. 55:242-249.

28. Sibson, N.R., et al. 2002. TNF-alpha reduces cerebral blood volume and disrupts tissue homeostasis via an endothelin- and TNFR2-dependent pathway. Brain. 125:2446-2459.

29. McAteer, M.A., et al. 2007. Magnetic resonance imaging of endothelial adhesion molecules in mouse atherosclerosis using dual-targeted microparticles of iron oxide. Arterioscler. Thromb. Vasc. Biol. 28:77-83.

30. Bell, M.D., and Perry, V.H. 1995. Adhesion molecule expression on murine cerebral endothelium following the injection of a proinflammagen or during acute neuronal degeneration. J. Neurocytol. 24:695-710.

31. McHale, J.F., Harari, O.A., Marshall, D., and Haskard, D.O. 1999. Vascular endothelial cell expression of ICAM- 1 and VCAM- 1 at the onset of eliciting contact hypersensitivity in mice: evidence for a dominant role of TNF-alpha. J. Immunol. 162:1648-1655.

32. Proescholdt, M.G., et al. 2002. Intracerebroventricular but not intravenous interleukin-1beta induces widespread vascular-mediated leukocyte infiltration and immune signal mRNA expression followed by brain-wide glial activation. Neuroscience.
112:731-749

33. Carvalho-Tavares, J., et al. 2000. A role for platelets and endothelial selectins in tumor necrosis factoralpha-induced leukocyte recruitment in the brain microvasculature. Circ. Res. 87:1141-1148.

34. Bernardes-Silva, M., Anthony, D.C., Issekutz, A.C., and Perry, V.H. 2001. Recruitment of neutrophils across the blood-brain barrier: the role of E- and P-selectins. J. Cereb. Blood Flow Metab. 21:1115-1124.

35. Blamire, A.M., et al. 2000. Interleukin-1beta induced changes in blood-brain barrier permeability, apparent diffusion coefficient, and cerebral blood volume in the rat brain: a magnetic resonance study. J. Neurosci. 20:8153-8159.

36. Schwarz, M., et al. 2004. Reversibility versus persistence of GPIIb/IIIa blocker-induced conformational change of GPIIb/IIIa (alphaIIbbeta3, CD41/ CD61). J. Pharmacol. Exp. Ther. 308:1002-1011.

37. Wilcockson, D.C., Campbell, S.J., Anthony, D.C., and Perry, V.H. 2002. The systemic and local acute phase response following acute brain injury. J. Cereb. Blood Flow Metab. 22:318-326.

38. Blond, D., et al. 2002. Differential induction of interleukin-1beta and tumour necrosis factoralpha may account for specific patterns of leukocyte recruitment in the brain. Brain Res. 958:89-99.

39. Andersson, P.B., Perry, V.H., and Gordon, S. 1992. The acute inflammatory response to lipopolysaccharide in CNS parenchyma differs from that in other body tissues. Neuroscience. 48:169-186.

40. Matyszak, M.K., and Perry, V.H. 1995. Demyelination in the central nervous system following a delayed-type hypersensitivity response to bacillus Calmette-Guerin. Neuroscience. 64:967-977.

41. Anthony, D., et al. 1998. CXC chemokines generate age-related increases in neutrophil-mediated brain inflammation and blood-brain barrier breakdown. Curr. Biol. 8:923-926. 